\title{
International Myeloma Working Group Standard Response Criteria
}

National Cancer Institute

\section{Source}

National Cancer Institute. International Myeloma Working Group Standard Response

Criteria. NCl Thesaurus. Code C159804.

Uniform consensus criteria created by the International Myeloma Working Group to describe response to treatment. 